\title{
Acometimento ocular em pacientes com AIDS e toxoplasmose do sistema nervoso central - antes e depois do HAART
}

\author{
Ocular involvement in AIDS patients with central nervous system toxoplasmosis - \\ before and after HAART
}

\author{
Moysés Zajdenweber ${ }^{1}$ \\ Cristina Muccioli² \\ Rubens Belfort $\mathbf{J r}^{3}$
}

\begin{tabular}{|l|}
\hline RESUMO \\
\hline Objetivo: Descrever o comprometimento ocular em pacientes com AIDS \\
e toxoplasmose do sistema nervoso central, comparando os períodos pré \\
e pós-terapia anti-retroviral (HAART). Métodos: Em estudo retrospectivo, \\
comparamos 118 pacientes com AIDS e toxoplasmose do sistema nervo- \\
so central que foram examinados em nossa instituição antes do pré e pós- \\
terapia anti-retroviral(1994-1996) com 24 pacientes com AIDS e toxoplas- \\
mose do sistema nervoso central que foram tratados com pré e pós- \\
terapia anti-retroviral (1996-1999). Todos os pacientes foram submetidos \\
a exame oftalmológico completo e a testes específicos para confirmar o \\
diagnóstico e não houve interseção entre os grupos. Resultados: No \\
grupo pré e pós-terapia anti-retroviral, foi encontrado 23\% de toxoplas- \\
mose ocular entre os pacientes com toxoplasmose do sistema nervoso \\
central. O envolvimento ocular foi bilateral em 37\% dos casos. No grupo \\
dos pacientes que receberam pré e pós-terapia anti-retroviral, que tinham \\
CD4 médio de 256 cel/mm e carga viral média de 52.620 cópias, 16,6\% \\
apresentaram concomitantemente toxoplasmose ocular e toxoplasmose \\
do sistema nervoso central. O envolvimento ocular foi bilateral em 50\% \\
dos casos. Conclusão: Pacientes com AIDS e toxoplasmose do sistema \\
nervoso central apresentam freqüente associação com toxoplasmose \\
ocular. Embora a incidência de infecções oportunistas tenha diminuído \\
desde a introdução de pré e pós-terapia anti-retroviral, a toxoplasmose \\
ocular continua sendo freqüente em pacientes com toxoplasmose do \\
sistema nervoso central.
\end{tabular}

Descritores: Toxoplasmose ocular; Retinite por citomegalovírus; Doenças do sistema nervoso central; Infeções oportunísticas relacionada a AIDS; Síndrome da imunodeficiência adquirida; Terapia anti-retroviral de alta atividade

\section{INTRODUÇ̃̃̃O}

O Toxoplasma gondii é um importante agente patogênico que pode causar doença sistêmica e ocular em indivíduos imunocompetentes e imunodeficientes $^{(1)}$. A toxoplasmose pode ser uma doença agressiva e freqüentemente fulminante em indivíduos com a síndrome da imunodeficiência adquirida (AIDS), causando infecção do sistema nervoso central, infecção visceral e dos nódulos linfáticos ${ }^{(2)}$. Com o crescente número de descrições de lesões retinianas associadas com AIDS, a toxoplasmose vem se mostrando uma importante causa de retinite infecciosa ${ }^{(3)}$. 
A toxoplasmose do sistema nervoso central é a causa mais freqüente de lesão cerebral focal em pacientes com $\operatorname{AIDS}^{(1)}$. Embora a encefalite por toxoplasmose seja comum na AIDS, o envolvimento ocular é considerado raro $^{(2)}$. Lesões oculares podem ser a primeira manifestação de doença intracraniana ou disseminada, porém podem ocorrer sem evidências de envolvimento intracraniano ${ }^{(4)}$.

A toxoplasmose ocular caracteriza-se por lesão exsudativa focal que secundariamente pode afetar a coróide e o segmento anterior levando à baixa de acuidade visual. Toxoplasmose ocular é normalmente uma doença de acometimento unilateral, porém em indivíduos imunodeprimidos o acometimento pode ser bilateral. Nestes indivíduos a lesão retiniana é extensa e pode levar a importante acometimento da função visual se não tratada ${ }^{(5)}$.

Um grande número de mudanças se estabeleceu em relação às infecções oportunistas secundárias, sistêmicas e oculares, em pacientes com AIDS com o advento da terapia anti-retroviral (HAART), especialmente em relação a retinite por citomegalovírus (CMV).

Após a introdução do HAART, uma diminuição importante na incidência e na severidade das infecções oportunistas secundárias oculares como a retinite por CMV e a toxoplasmose ocular foram observadas.

Neste trabalho avaliamos em estudo retrospectivo as características da toxoplasmose ocular em pacientes com AIDS no período pré e pós HAART.

\section{MÉTODOS}

Um total de 142 prontuários de pacientes com AIDS e toxoplasmose do sistema nervoso central (critérios CDC) foram examinados no Departamento de Oftalmologia da Universidade Federal de São Paulo - Escola Paulista de Medicina. Do total de prontuários avaliados 118 eram de pacientes examinados no período pré HAART (1994-96) e 24 do período pós HAART (1996-99).

Todos os pacientes tinham sorologia positiva para HIV, determinada por testes ELISA (enzyme-linked immunosorbent assay) e Western blot e sorologia positiva para toxoplasmose (IgG).

A retinite por toxoplasmose foi diagnosticada em pacientes que apresentavam lesão ativa ou inativa de retinite baseada em seu aspecto clínico e afastado os diagnósticos diferenciais como CMV, sífilis e herpes.

O exame oftalmológico incluiu medida de acuidade visual, biomicroscopia, oftalmoscopia indireta e retinografia se necessário.

O diagnóstico de encefalite por toxoplasmose foi realizado por neurologista, baseado em sinais e sintomas de envolvimento do sistema nervoso central e por lesões intracranianas detectadas por tomografia computadorizada ou ressonância nuclear magnética.

Nenhum paciente foi incluído nos 2 grupos, pré e pós HAART.

Todos os pacientes foram tratados com a combinação de pirimetamina $25 \mathrm{mg} /$ dia, sulfadiazina $4 \mathrm{~g} /$ dia e ácido folínico $3 \mathrm{x}$ semana, por 40 dias.

\section{RESULTADOS}

Dos 118 pacientes com toxoplasmose do sistema nervoso central que não foram tratados com HAART, o exame oftalmológico mostrou que 71 pacientes (60\%) não apresentavam acometimento ocular; 27 (23\%) apresentaram lesões retinocoroidianas características de toxoplasmose ocular; 22 (19\%) retinite por CMV; 8 (6,7\%) exsudatos algodonosos; $8(6,7 \%)$ atrofia óptica; $5(4,2 \%)$ edema de papila; $3(2,5 \%)$ retinite herpética e $2(1,6 \%)$ papilite (Tabela 1$)$.

Em 3 pacientes, retinite por CMV ativa e toxoplasmose ocular ativa estavam presentes no mesmo olho. Dois pacientes apresentaram cicatriz de toxoplasmose em um olho e retinite por CMV ativa no olho contralateral.

A toxoplasmose ocular era bilateral em $44(37,2 \%)$ pacientes.

Entre os 27 pacientes com toxoplasmose ocular e do sistema nervoso central concomitantes, 55\% apresentavam lesão de retinocoroidite ativa.

Onze pacientes $(9,3 \%)$ desenvolveram novas lesões de retinocoroidite por toxoplasmose durante o tratamento com sulfadiazina e pirimetamina para toxoplasmose do sistema nervoso central.

Dos 24 pacientes em uso de HAART que apresentavam toxoplasmose do sistema nervoso central o exame oftalmológico mostrou que 9 (37,5\%) não apresentavam comprometimento ocular; $4(16,6 \%)$ apresentavam lesões retinocoroidianas características de toxoplasmose ocular; $3(12,5 \%)$ com retinite por CMV; 2 (8,3\%) com descolamento de retina; 2 $(8,3 \%)$ com retinite herpética; 2 (8,3\%) com membrana epiretiniana; $1(4,2 \%)$ com atrofia óptica; $1(4,2 \%)$ com papiledema; 1 $(4,2 \%)$ com papilite; $1(4,2 \%)$ com buraco macular e $1(4,2 \%)$ com vasculite (Tabela 2 ).

Entre os 4 pacientes com toxoplasmose ocular concomitante com acometimento de sistema nervoso central, 1 apresentava lesão ativa de retinocoroidite e cicatrizes e 3 somente cicatrizes.

No grupo que recebeu HAART o CD4 médio era de $256 \mathrm{cel} /$ $\mathrm{mm}^{3}$ e a carga viral média era de 52.620 cópias.

Tabela 1. Achados oftalmológicos no período pré-HAART

Lesões oculares

Sem acometimento ocular

Toxoplasmose ocular

Retinite por CMV

Exsudatos algodonosos

Atrofia óptica

Papiledema

Retinite herpética

Papilite
Freqüência

$60,0 \%$ (71 pacientes) $23,0 \%$ (27 pacientes)

$19,0 \%$ (22 pacientes)

$6,7 \%$ ( 8 pacientes)

$6,7 \%$ ( 8 pacientes)

$4,2 \%$ ( 5 pacientes)

$2,5 \%$ ( 3 pacientes)

$1,6 \%$ ( 2 pacientes) 


\begin{tabular}{|c|c|}
\hline Lesões oculares & Freqüência \\
\hline Sem acometimento ocular & $37,5 \%$ (9 pacientes) \\
\hline Toxoplasmose ocular & $16,6 \%$ (4 pacientes) \\
\hline Retinite por CMV & $12,5 \%$ (3 pacientes) \\
\hline Retinite herpética & $8,3 \%$ (2 pacientes) \\
\hline Membrana epiretiniana & $8,3 \%$ (2 pacientes) \\
\hline Atrofia óptica & $4,2 \%$ (1 paciente) \\
\hline Papiledema & $4,2 \%$ (1 paciente) \\
\hline Papilite & $4,2 \%$ (1 paciente) \\
\hline Buraco macular & $4,2 \%$ (1 paciente) \\
\hline Vasculite & $4,2 \%$ (1 paciente) \\
\hline
\end{tabular}

\section{DISCUSSÃO}

A toxoplasmose do sistema nervoso é comum na AIDS e o envolvimento ocular apesar de ser menos freqüente é uma entidade importante por ser uma causa tratável que pode levar a importante perda visual ${ }^{(5)}$. Lesões oculares podem ser a primeira manifestação de toxoplasmose intracraniana ou disseminada ou podem ocorrer sem evidências de doença intracraniana ${ }^{(5)}$.

Pacientes com AIDS e toxoplasmose do sistema nervoso central apresentam uma freqüente associação com toxoplasmose ocular. Embora a incidência de infecções oportunistas tenha diminuído com a introdução das drogas anti-retrovirais a toxoplasmose ocular continua a ser freqüente em pacientes com toxoplasmose do sistema nervoso central.

Em nosso trabalho comparando os grupos pré e pós HAART observamos que houve uma diminuição na freqüência de infecções oportunistas como a retinite por CMV e a retinite herpética naqueles pacientes que receberam o HAART, entretanto este fato não foi observado quando comparamos os dois grupos em relação à toxoplasmose.

\section{CONCLUSÃO}

Pacientes com AIDS e toxoplasmose do sistema nervoso central apresentam uma freqüente associação com toxoplasmose ocular. Embora a incidência de infecções oportunistas tenha diminuído desde a introdução do HAART a toxoplasmose ocular continua sendo freqüente em pacientes com toxoplasmose do sistema nervoso central.

\section{ABSTRACT}

Purpose: To describe the ophthalmologic involvement in patients with Acquired Immunodeficiency Syndrome - AIDS and central nervous system toxoplasmosis comparing the period before and after highly active antiretroviral therapy (HAART). Methods: In a retrospective study, we compared 118 Acquired
Immunodeficiency Syndrome patients with central nervous system toxoplasmosis who were examined at our institution before highly active antiretroviral therapy (from 1994 - 1996) with 24 patients with Acquired Immunodeficiency Syndrome and central nervous system toxoplasmosis who were being treated with highly active antiretroviral therapy (from 1996 1999). All patients were submitted to a complete ophthalmological examination as well as specific tests to confirm the diagnosis and there was no intersection between the groups. Results: In the pre- highly active antiretroviral therapy group, it was found that $23 \%$ of the patients with central nervous system toxoplasmosis had also ocular toxoplasmosis. Ocular involvement was bilateral in $37.2 \%$ of the cases. In the group of patients receiving highly active antiretroviral therapy, who had an average CD4 of 256 cells $/ \mathrm{mm}^{3}$ and average of viral load of 52,620 copies, $16.6 \%$ had concomitant ocular and central nervous system toxoplasmosis. Ocular involvement was bilateral in $50 \%$ of the cases. Conclusions: Acquired Immunodeficiency Syndrome patients with central nervous system toxoplasmosis have a frequent association with ocular toxoplasmosis. Although the incidence of opportunistic infections has decreased since the introduction of recent antiretroviral therapeutic strategies ocular toxoplasmosis continues to be frequent in patients with cen.ral nervous system toxoplasmosis.

Keywords: Toxoplasmosis, ocular; Cytomegalovirus retinitis; Central nervous system diseases; AIDS-related opportunistic infection; Acquired immunodeficiency syndrome; Antiretroviral therapy, highly active

\section{REFERÊNCIAS}

1. Nussenblatt RB, Belfort Jr. R. Pathogenesis of Inflammatory conditions of the retina: Toxoplasmic Retinochoroiditis. 2th ed. In: Tabbara KF, Hyndiuk R. Infections of the eye. Little, Brown; 1996. p.61-5.

2. Perkins ES. Uveitis and toxoplasmosis. New York: Little, Brown; 1961.

3. Silveira C, Belfort Jr. R, Burnier M, Nussenblatt RB. Acquired toxoplasmosis infection as the cause of toxoplasmic retinochoroiditis in families. Am $\mathrm{J}$ Ophthalmol. 1988;106(3):362.

4. Holland GN, O'Connor GR, Belfort Jr R, Remington JS. Toxoplasmosis. In: Pepose JS, Holland GN, Wilhelmus KR. Ocular infection and immunity. Philadelphia: Mosby; 1995. p.183-223.

5. Holland GN, O'Connor GR, Diaz RF, Minasi P, Wara WM. Ocular toxoplasmosis in immunossupressed nonhuman primates. Invest Ophthalmol Vis Sci. 1988;29(6):835-42.

6. Whitcup SM. Cytomegalovirus retinitis in the era of highly active antiretroviral therapy. JAMA. 2000;283(5):653-7.

7. Abreu MT, Belfort Jr R, Oréfice F. Toxoplasmose ocular. In: Oréfice F, Belfort Jr R. Uveítes. São Paulo: Roca; 1987. p.211-30.

8. Muccioli C, Belfort Junior R, Lottenberg C, Lima J, Santos P, Kim M, et al. Achados oftalmológicos em AIDS: avaliação de 445 casos atendidos em 1 ano. Rev Assoc Med Bras. 1994;40(3):155-8.

9. Duarte V, Tavares W, Ribeiro MP, Chimelli LC, Hahn MY. Comprometimento neurológico por toxoplasmose em pacientes com AIDS. DST J Bras Doenças Sex Transm. 1997;9(5):4-9.

10. Dalston MO, Tavares W, Hahn MD, Mello WA, Bedran M, Lessa MA, et al. Neurotoxoplasmose em pacientes com síndrome de imunodeficiência adquirida; estudo de 33 casos. Rev Bras Neurol. 1996;32(4):133-41. 